\title{
Genetic characterization of canine parvovirus from dogs in Pakistan
}

\author{
M. Z. SHABBIR ${ }^{1 *}$, M. U. SOHAIL ${ }^{2}$, U. N. CHAUDHARY ${ }^{3}$, W. YAQUB ${ }^{1}$, I. RASHID ${ }^{1}$, M. H. SALEEM ${ }^{1}$, M. MUNIR $^{4}$
}

${ }^{1}$ University of Veterinary and Animal Sciences, Lahore, Pakistan; ${ }^{2}$ Government College University, Faisalabad, Pakistan; ${ }^{3}$ The Roslin Institute, University of Edinburgh, Scotland, UK; ${ }^{4}$ The Pirbright Institute, UK

Received June 20, 2016; revised August 31, 2016; accepted March 16, 2017

\begin{abstract}
Summary. - Canine parvoviruses (CPV) exist as antigenic variants with varying frequencies and genetic variabilities across the globe. Given the endemicity and high prevalence in Pakistan, we characterized the CPVs originating from dogs-population to elucidate viral diversity and evolution. Fecal samples from clinically diseased pups ( $n=17)$ of different breeds and age (2-6 months) were processed for hemagglutination assay (HA), and later for partial amplification of VP2 gene sequence and amino acid analysis. A total of 11 samples (64.71\%) were found positive both in hemagglutination and PCR assays. Phylogenetic and evolutionary analysis demonstrated higher genetic heterogeneity in studied strains and constituted seven clusters within the CPV-2a group, however, they shared high level of identity with Chinese strains. Further studies are necessary to elucidate genetic analysis and epidemiology of $\mathrm{CPV}$ variants across a wide geographical area of the country.
\end{abstract}

Keywords: canine parvovirus; dog; CPV-2a; Pakistan

\section{Introduction}

Canine parvovirus (CPV) enteritis is the major viral infection of young puppies characterized by intestinal hemorrhages and bloody diarrhea (Lin et al., 2014). The virus belongs to the family Parvoviridae and has a non-enveloped, single-stranded linear deoxyribonucleic acid of approximately $5 \mathrm{~kb}$ in length. The genome consists of two major open reading frames (ORFs); first ORF encodes for two non-structural proteins (NS1 and NS2) whereas the second ORF encodes for two capsid proteins (VP1 and VP2). A third protein, known as VP3, is a proteolytic by-product of VP2 (Reed et al., 1988; Agbandje et al., 1995).

With the first identification in 1978 (Appel et al., 1979), the virus has known to occur in two distinct types as CPV-1 and CPV-2 based upon antigenic differences. Of these, $\mathrm{CPV}-2$ exhibited enteric and respiratory symptoms in puppies. Soon after the emergence of CPV-2, minor amino acid substitutions have caused appearance of new antigenic types

E-mail: shabbirmz@uvas.edu.pk; phone: +92-42-99210256. Abbreviations: $\mathrm{CPV}=$ canine parvovirus; $\mathrm{HA}=$ hemagglutination assay; $\mathrm{mAbs}=$ maternal antibodies
(CPV-2a and CPV-2b) around 1980s (Parrish et al., 1991). From year 2000, a new antigenic variant (CPV-2c) has been identified in Europe (Buonavoglia et al., 2001; Decaro et al., 2007a), United States (Hong et al., 2007) and South America (Perez et al., 2007). Hence, with the passing of time, different antigenic variants are being reported from epidemics in canine population across the globe, replacing original CPV-2 (Parrish et al., 1991; Buonavoglia et al., 2001; Decaro et al., 2007a; Lin et al., 2014). This evolutionary potential of CPV raises concerns not only about the vaccine strains used but also necessitates continuous surveillance to understand the genetics of circulating strains.

In Pakistan, based upon clinical diagnostics of canine parvovirus enteritis, an overall prevalence of $21-22.7 \%$ has been recorded that varied with age, breed and sex (Jafri and Rabbani, 1999; Towakal et al., 2010; Khan et al., 2006; Umar et al., 2015). Although, modified live vaccine is used to vaccinate the pups, disease occurrence even in vaccinated pups is not uncommon. Besides concerns about variations in pups' genetics to develop vaccine-induced immunity (Buonavoglia et al., 2001) and interference of maternal antibodies (mAbs) against active immune response at an early age (Larson and Schultz, 1997), a continuous evaluation of circulating field variants, appropriate diagnostic 
and control strategies are needed. Nevertheless, despite high prevalence and endemicity, there lacks genetic analysis of prevailing genotypes or variants in Pakistan. Since emerging and widely spread CPV-2 has considerable and invariable evolutionary relevance across the globe, we sequenced VP2 gene of CPV from clinical samples of suspected dogs followed by sequence and phylogenomic analysis. The obtained information provides an insight towards genetic diversity of indigenous strains and their comparative evolution to those reported globally.

\section{Materials and Methods}

Sample collection. Rectal swabs $(\mathrm{n}=17)$ were collected from owned puppies originating from different areas in and around Lahore district, Punjab province, Pakistan. The district is situated 217 $\mathrm{m}$ above sea level, lies between $31^{\circ} 15^{\prime}-31^{\circ} 45^{\prime} \mathrm{N}$ and $74^{\circ} 01^{\prime}-74^{\circ} 39^{\prime}$ $\mathrm{E}$ and covers a total land area of $1,772 \mathrm{~km}^{2}$. Human population exceeds 10 million heads. The affected pups were brought to private and a public veterinary clinic at the University of Veterinary and Animal Sciences, Lahore. The puppies had symptoms suggestive of CPV enteritis such as off-feed, emaciation, dehydration, fever, vomiting and bloody diarrhea. The affected animals were 2-6 months old and were from different breeds of pet dogs owned by residents in the district Lahore. Complete information regarding their age, sex, breed, vaccination status and clinical symptoms is given in Table 1.

Haemagglutination test. The collected swabs were diluted with a ratio of 1:5 using sterile normal saline solution $(0.85 \% \mathrm{NaCl})$ and centrifuged for $10 \mathrm{~min}$ at $1000 \times \mathrm{g}$. The supernatant of each sample was passed through $0.22 \mu \mathrm{m}$ syringe filter (Millipore, USA) and processed for hemagglutination assay (HA) (Carmichael et al., 1980). Only the HA positive samples $(n=11)$ were processed further for PCR, sequencing and subsequent phylogenetic analysis. Rectal swabs from clinically healthy pups were taken as negative controls whereas commercially available vaccine was used as positive control in both assays, the HA and PCR.

Genome extraction and PCR amplification. Supernatants from the samples that appeared positive in HA were adsorbed on the FTA QIAcard (QIAGEN, Germany) and were shipped to Swedish University of Agricultural Sciences, Uppsala, Sweden for processing. The genetic material was eluted from four $3 \mathrm{~mm}$ punches using DNA elution buffers and stored at $-20^{\circ} \mathrm{C}$ until use. The extracted DNA was then subjected to amplification of gene encoding capsid protein (VP2) using a forward primer (3556-3575, 5'-CAGG TGATGAATTTGCTACA-3') and a reverse primer (4185-4166, 5'-CATTTGGATAAACTGGTGGT-3') that encompass important codon residues of biological and epidemiological relevance. The thermal conditions and reaction protocol was strictly followed as described previously (Buonavoglia et al., 2001). The amplification was confirmed by electrophoresis with a $2 \%$ agarose gel in $1 \mathrm{x}$ Tris acetate EDTA (TAE) buffer. Amplified fragments were excised from the gel and purified using PCR clean-up wizard (Promega Co., USA) and were processed for sequencing using ABI PRISM BigDye Terminator version 3.1 (Applied Biosystems, USA), according to the manufacturer's instructions. Same PCR primers were used to sequence the products and each DNA fragment was sequenced at least twice in both directions.

Sequence and phylogenetic analysis. Sequence assembly and editing were performed using the SEQMAN program from DNASTAR Lasergene suite 9 (version 9.0.4 39; DNASTAR, Inc., USA). The unambiguous and consensus sequence generated from each contig was analysed through BLAST (Basic Local Alignment Search Tool) program. The percent similarity were used as benchmark of the genotype and genetic relatedness. The representative sequences were downloaded from the NCBI database and were aligned with the studied sequences in the BioEdit using ClustalW algorithm. The aligned dataset including publicly available representatives were processed in MEGA6 and Neighbor Joining tree $(\mathrm{NJ}$ tree) was constructed (bootstrap replicates $=1000$; seed $=64,248)$ using Kimura 2 parameter method for pairwise deletion at uniform rates.

All the obtained sequences were submitted to GenBank and are available under Acc. Nos. KU248465-KU248475.

\section{Results}

We processed feces from 17 suspected dogs for HA test using 2-fold dilutions. Eleven of tested samples showed titer ranging from 1:64 to 1:256. The remaining samples showed titer $\leq 32$. All the HA positive dogs $(n=11)$ were between 2-6 months of age with almost an equal distribution of sex (male, 5: female, 6). Nine of them were purebred while two were non-descript/unknown. Except three pups, all had the history of vaccination. History of dam vaccination was available for two pups only, while none of the owner had the antibody titer-record. Anorexia, vomiting, hemorrhagic gastroenteritis and dehydration were the common clinical symptoms to all pups (Table 1).

Given the well-known criteria, nucleotide sequence at $4062-4064$ of full genome and the corresponding $426^{\text {th }}$ codon of the VP2 gene was used to classify CPV strains into different groups compared to reference strains. All the studied CPVs corresponded to characteristic nucleotide sequence (AAT) and subsequent amino acid substitution ( $\mathrm{N}$, asparagine) corresponding to antigenic variant CPV-2a. Varying in different studied dogs in nucleotide and subsequent codon profile, various synonymous and two non-synonymous substitutions were identified within CPV-2a variant. Each of five of the studied strain had the non-synonymous substitution located at codon 267 and 440. The reference codon F (Phe, TTT) was replaced by Y (Tyr, TAT) at codon 267 while T (ACA, Thr) was replaced by A (GCA, Ala) at codon 440 (Table 2). 
Table 1. A brief history of clinical samples used in this study

\begin{tabular}{|c|c|c|c|c|c|c|}
\hline Dog breed & $\begin{array}{l}\text { Sampling } \\
\text { (month, year) }\end{array}$ & $\begin{array}{c}\text { Age } \\
\text { (months) }\end{array}$ & Sex & Clinical symptoms & Vaccination & HA titre \\
\hline Unknown & February, 2011 & 5 & Male & $\mathrm{A}, \mathrm{C}, \mathrm{D}, \mathrm{H}, \mathrm{I}, \mathrm{J} \mathrm{K}, \mathrm{L}, \mathrm{M}$ & Yes & $1: 64$ \\
\hline German Shepherd & February, 2011 & 4 & Male & A,B,D,E,F,H,J, K,L & Yes & $1: 128$ \\
\hline Unknown & March, 2011 & 5 & Female & A,C,D,E,F,G,H,I,J, K,L,M & Yes & $1: 128$ \\
\hline German Shepherd & March, 2011 & 5 & Female & $\mathrm{A}, \mathrm{B}, \mathrm{D}, \mathrm{F}, \mathrm{H}, \mathrm{I}, \mathrm{J}, \mathrm{K}, \mathrm{M}$ & No & $1: 64$ \\
\hline German Shepherd & December, 2011 & 4 & Female & A,B,E,H,J, K,L,M & Yes & $1: 64$ \\
\hline German Shepherd & January, 2012 & 3 & Male & A,B,C,E,F,G,H,I,J K & No & $1: 64$ \\
\hline Bulldog & January, 2012 & 4 & Female & A,D,E,F,H,J, K,L & Yes & $1: 256$ \\
\hline Poodle & January, 2012 & 4 & Female & A,F,G,H,I,J K & Yes & $1: 64$ \\
\hline Bulldog & February, 2012 & 2 & Male & $\mathrm{A}, \mathrm{C}, \mathrm{D}, \mathrm{H}, \mathrm{I}, \mathrm{J} \mathrm{K}, \mathrm{M}$ & No & $1: 64$ \\
\hline German Shepherd & April, 2012 & 6 & Male & A,B,D,H,I,J, K,M & Yes & $1: 128$ \\
\hline German Shepherd & April, 2012 & 5 & Female & A,B,E,F,H,I,J, K,L,M & Yes & $1: 128$ \\
\hline
\end{tabular}

$\mathrm{A}=$ anorexia; $\mathrm{B}=$ hypothermia; $\mathrm{C}=$ tachypnea; $\mathrm{D}=$ congested mucus membrane; $\mathrm{E}=$ drooling of saliva; $\mathrm{F}=$ ventro-lateral flexion of neck; $\mathrm{G}=$ lateral recumbancy; $\mathrm{H}$ = vomiting; $\mathrm{I}=$ dull mentation; $\mathrm{J}=$ hemorrhagic gastroenteritis; $\mathrm{K}=$ dehydration; $\mathrm{L}=$ retching; $\mathrm{M}=$ foul-smelled diarrhea.

The sequence specificity of CPV strains was investigated using BLAST. Based on the query coverage and percentage identity, all sequences showed high specificity to CPV strains available in GenBank database. BLAST analysis of CPV sequences revealed that the CPV sequence of 9 Pakistani strains had maximum identity (100\%) with CPV-SD-14-7 (KR611517), CPV/BJ450 (KR869668), and CPV/CN/JL5/2013 (KR002798). Two strains characterized from Pakistan showed 99\% identity with the CPV-SD-14-7 (KR611517) and CPV/ CN/JL5/2013 (KR002798). Interestingly, all these top hits were recently (2013-2015) characterized from China, a country that shares border with Pakistan. A broad-scale phylogenetic analysis of CPV strains with representative VP2 genes demonstrated the clustering into three broad groups, $2 \mathrm{a}, 2 \mathrm{~b}$ and $2 c$ (Fig. 1). The group $2 \mathrm{a}$ appeared to be the most widely described and geographically diverse. All Pakistani CPV strains were grouped as CPV 2a with dispersed distribution. A highresolution phylogenetic tree (Fig. 2), only consisting of CPV strains from $2 \mathrm{a}$, further clarified that all 11 strains constituted a total of 7 discrete clusters within 2a group.

Table 2. Pattern of amino acid variations in the VP-2 gene compared to reference strains of CPV-2 variants

\begin{tabular}{|c|c|c|c|c|c|c|c|c|c|c|c|c|c|c|}
\hline \multirow{2}{*}{ Strain } & \multirow{2}{*}{ Origin and year } & \multirow{2}{*}{ Acc. No. } & \multicolumn{11}{|c|}{ Amino Acid position } & \multirow{2}{*}{ Genotype } \\
\hline & & & 267 & 297 & 300 & 305 & 367 & 426 & 427 & 436 & 440 & 445 & 450 & \\
\hline \multicolumn{15}{|l|}{ Reference strains } \\
\hline CPV-N & USA, 1988 & M19296 & $\mathrm{F}$ & $\mathrm{S}$ & A & $\mathrm{D}$ & $\mathrm{Y}$ & $\mathbf{N}$ & $\mathrm{D}$ & I & $\mathrm{T}$ & $\mathrm{T}$ & $\mathrm{T}$ & 2 \\
\hline CPV-V120 & Vietnam, 2000 & AB054215 & $\mathrm{F}$ & A & G & $\mathrm{Y}$ & $\mathrm{D}$ & $\mathbf{N}$ & $\mathrm{D}$ & I & $\mathrm{T}$ & $\mathrm{T}$ & $\mathrm{T}$ & $2 \mathrm{a}$ \\
\hline CPV-39 & USA,1984 & M74849 & $\mathrm{F}$ & $\mathrm{S}$ & G & $\mathrm{Y}$ & $\mathrm{D}$ & $\mathbf{D}$ & $\mathrm{D}$ & I & $\mathrm{T}$ & $\mathrm{T}$ & $\mathrm{T}$ & $2 b$ \\
\hline CPV-G7/97 & Germany,1997 & FJ005196 & $\mathrm{F}$ & A & G & $\mathrm{Y}$ & $\mathrm{D}$ & $\mathbf{E}$ & $\mathrm{D}$ & I & $\mathrm{T}$ & $\mathrm{T}$ & $\mathrm{T}$ & $2 c$ \\
\hline \multicolumn{15}{|c|}{ Pakistani strain identified from clinical samples } \\
\hline German Shepherd & 2011 & KU248465 & $\mathrm{F}$ & A & G & $\mathrm{Y}$ & $\mathrm{D}$ & $\mathbf{N}$ & $\mathrm{D}$ & I & $\mathrm{T}$ & $\mathrm{T}$ & $\mathrm{T}$ & \\
\hline Poodle & 2012 & KU248466 & $\mathrm{F}$ & A & G & $\mathrm{Y}$ & $\mathrm{D}$ & $\mathbf{N}$ & $\mathrm{D}$ & I & A & $\mathrm{T}$ & $\mathrm{T}$ & \\
\hline German Shepherd & 2011 & KU248467 & $\mathbf{Y}$ & $\mathrm{A}$ & G & $\mathrm{Y}$ & $\mathrm{D}$ & $\mathbf{N}$ & $\mathrm{D}$ & I & $\mathbf{A}$ & $\mathrm{T}$ & $\mathrm{T}$ & \\
\hline Bulldog & 2012 & KU248468 & $\mathbf{Y}$ & A & G & $\mathrm{Y}$ & $\mathrm{D}$ & $\mathbf{N}$ & $\mathrm{D}$ & I & $\mathbf{A}$ & $\mathrm{T}$ & $\mathrm{T}$ & \\
\hline Bulldog & 2012 & KU248469 & $\mathrm{F}$ & $\mathrm{A}$ & G & $\mathrm{Y}$ & $\mathrm{D}$ & $\mathbf{N}$ & $\mathrm{D}$ & I & $\mathrm{T}$ & $\mathrm{T}$ & $\mathrm{T}$ & \\
\hline Unknown/Non-descript & 2011 & KU248470 & $\mathrm{F}$ & $\mathrm{A}$ & G & $\mathrm{Y}$ & $\mathrm{D}$ & $\mathbf{N}$ & $\mathrm{D}$ & I & $\mathrm{T}$ & $\mathrm{T}$ & $\mathrm{T}$ & $2 \mathrm{a}$ \\
\hline Unknown/Non-descript & 2011 & KU248471 & $\mathbf{Y}$ & A & G & $\mathrm{Y}$ & $\mathrm{D}$ & $\mathbf{N}$ & $\mathrm{D}$ & I & $\mathbf{A}$ & $\mathrm{T}$ & $\mathrm{T}$ & \\
\hline German Shepherd & 2011 & KU248472 & $\mathbf{Y}$ & $\mathrm{A}$ & G & $\mathrm{Y}$ & $\mathrm{D}$ & $\mathbf{N}$ & $\mathrm{D}$ & I & $\mathrm{T}$ & $\mathrm{T}$ & $\mathrm{T}$ & \\
\hline German Shepherd & 2012 & KU248473 & $\mathrm{F}$ & A & G & $\mathrm{Y}$ & $\mathrm{D}$ & $\mathbf{N}$ & $\mathrm{D}$ & I & $\mathrm{T}$ & $\mathrm{T}$ & $\mathrm{T}$ & \\
\hline German Shepherd & 2012 & KU248474 & $\mathbf{Y}$ & A & G & $\mathrm{Y}$ & $\mathrm{D}$ & $\mathbf{N}$ & $\mathrm{D}$ & I & A & $\mathrm{T}$ & $\mathrm{T}$ & \\
\hline German Shepherd & 2012 & KU248475 & $\mathrm{F}$ & $\mathrm{A}$ & G & $\mathrm{Y}$ & $\mathrm{D}$ & $\mathbf{N}$ & $\mathrm{D}$ & I & $\mathrm{T}$ & $\mathrm{T}$ & $\mathrm{T}$ & \\
\hline
\end{tabular}




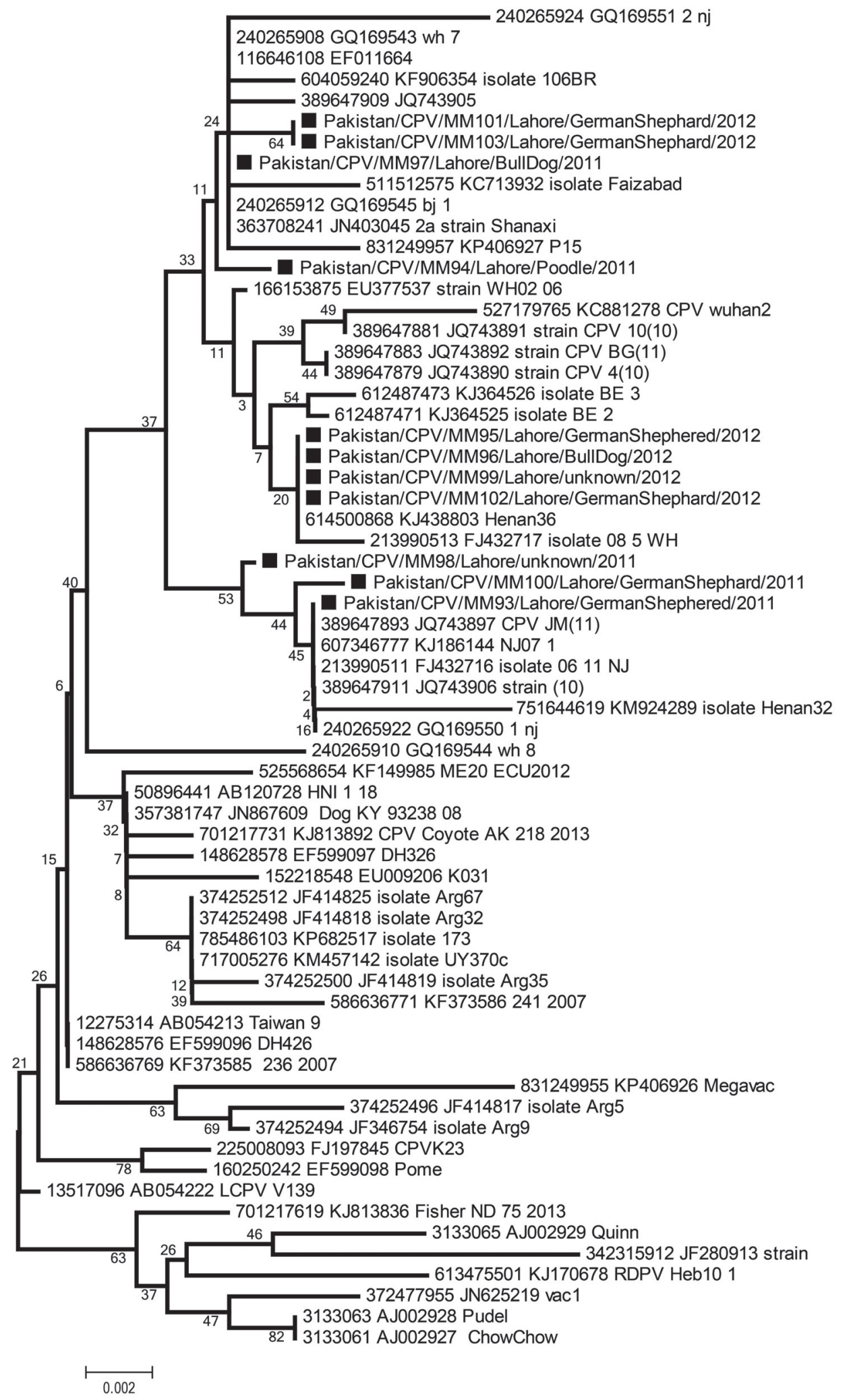

Fig. 1

Phylogenetic analysis of partial VP2 gene of CPVs

The nucleotide sequences (VP2 gene) of different strains were compared to those of previously reported strains classified as genotype $2 \mathrm{a}, 2 \mathrm{~b}$ and $2 \mathrm{c}$. The classification of studied strains is indicated ( $\mathbf{\square})$ in the phylogenetic consensus tree along with bootstrap values at each branch. 


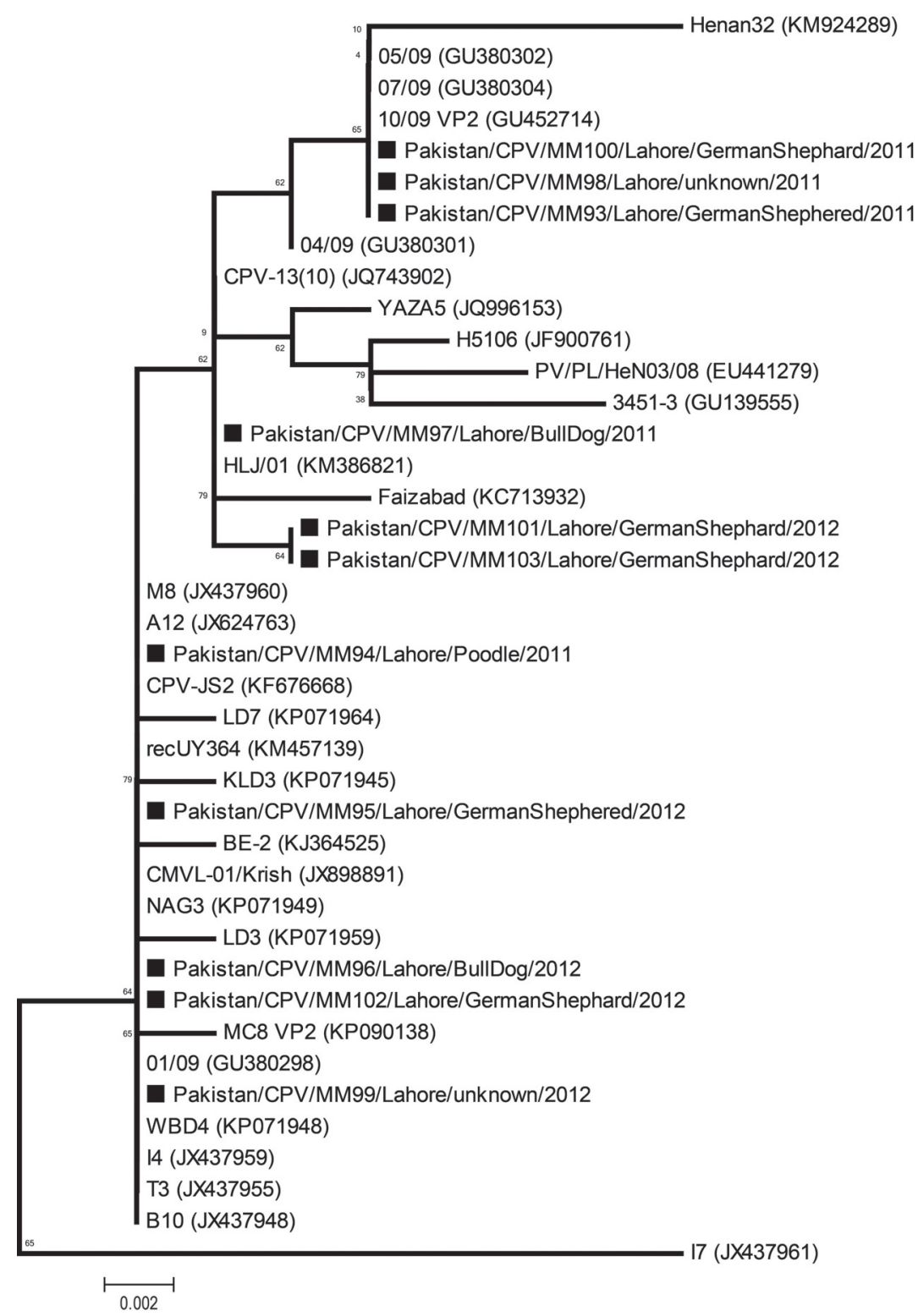

Fig. 2

Phylogenetic analysis of the VP2 gene of CPVs belonging to CPV-2a group

Strains studied in this study are indicated ( $\mathbf{\square})$. The scale indicates the number of substitutions per site.

\section{Discussion}

For the first time in Pakistan, we characterized the indigenous strains of CPV prevailing in dog population in the provincial capital of Punjab province. Sequence as well as amino acid based substitution(s) in the gene marker (VP2) have been widely applied to comprehend prevalent antigenic variants and their phylodynamics across different geographical areas (Shackelton et al., 2005; Martella et al., 2006; Nandi et al., 2010; Perez et al., 2012; Aldaz et al., 2013;
Zhao et al., 2015). Since clinical diagnostics is not always definite and symptoms with varying severity are associated with each variant, only rapid screening tests such as HA are being performed for identification of parvovirus in feces in Pakistan. We found $100 \%$ agreement for virus positivity in feces using HA and PCR assays. HA test is rapid, sensitive and easy to perform; however, viral agglutination to red blood cells varies with days post infection. It ranges from 128-1240 between 4-7 days or with onset of enteritis and it decreases within next two days (Carmichael et al., 1980). 
Currently, variation in incidence rate, sequence/amino acid composition and geography is being reported for CPV-2 and its variants CPV-2a, CPV-2b and CPV-2c worldwide (Decaro and Buonavoglia, 2012). However, we found CPV$2 \mathrm{a}$ as the sole prevalent variant in our samples from studied district. With difference in five residues in VP2 gene, CPV-2a is the first variant of CPV-2 that endured evolution while retaining several point mutations with unique ability to infect carnivores and cats (Truyen et al., 1996; Aldaz et al., 2013). Due to antigenic drift, it emerged in 1979 and, within one year, it spread worldwide replacing original CPV-2 (Nandi et al., 2010). Though a widespread prevalence of CPV-2c has been noted across several parts of the globe in the recent years, yet CPV-2a and $2 \mathrm{~b}$ are considered predominant variants in Asia (Lin et al., 2014). In a latest study conducted in India and China, few cases of CPV-2c were observed (Nandi et al., 2010; Zhao et al., 2015). Nevertheless, being a neighboring country to both India and China, we did not find CPV-2c in our clinical samples.

Variations within nucleotide and codon profile of CPV-2a variant were noted in some of our CPVs where two amino acids at codon 267 and 440 were replaced; F267Y and T440A. The biological role of codon 267 is still unknown while codon 440 is considered to be the major antigenic site that undergoes positive selection (Decaro et al., 2009). Three different codons at position 440 have been reported so far. These include original threonine residue and two substitutions caused by nucleotide transitions in the first position of the codon: (ACA $\rightarrow$ GCA) or Thr440Ala (Pereira et al., 2007; Maya et al., 2013; reported in this study too) and (ACA $\rightarrow$ TCA) or Thr440Ser (Aldaz et al., 2013). Despite being DNA viruses, CPVs show a high intrinsic substitution rate in their genome (Shackelton et al., 2005) leading to emergence and/or re-emergence of antigenic variants in susceptible population. Recently in Uruguay, an increased incidence of diverged CPV-2a strain has been reported that overwhelmed the former but predominant CPV-2c (Perez et al., 2012). These observations suggest the needs for continuous epidemiological surveillance to assess frequency and burden of prevailing variants and subsequent revision in diagnostics and application of homologous or heterologous vaccines for disease control.

Utilizing type-specific PCR, prevalence of CPV-2 and CPV-2b variants has been reported previously in Pakistan (Towakal et al., 2010). Since identification of prevailing variants can be assessed only with the sequence and amino acid substitution analysis, relying merely on product size could be misleading. This is important as commercial vaccines used in Pakistan contain attenuated CPV-2 and $2 \mathrm{~b}$ variants that have potential to be shed in feces (Decaro et al., 2007b; Meers et al., 2007) and, antigenic difference to each variant recognition is based upon single nucleotide difference at primer positions (4062 and 4449). Type $2 \mathrm{~b}$ specific primers used in a particular study (Pereira et al., 2000; Towakal et al., 2010) were with a nucleotide variation at the 3 ' end of the sense primer where $G \rightarrow A$ at nucleotide position 4062 is responsible for the change $\mathrm{D}(\mathrm{Asp}) 426 \mathrm{~N}$ (Asn) or CPV-2b to CPV-2a. Further, recently reported "new CPV-2a" variant has the same nucleotide at position 4449 that encodes Val-555 and this codon is common to CPV-2b. Further studies to re-evaluate dog population in Pakistan involving sequence and amino acid substitution analysis in a wider geographical area are much essential.

Two of the affected pups were from vaccinated dams, however, serological titers of vaccinates was not available due to lack of owner's awareness and immune titer evaluation facility in Pakistan. Since most of the studied dogs were purchased from kennels, owners were not aware of vaccination history as well as serological evaluation of dams. Further, except three dogs, all had the history of vaccination including two pups whose dams were vaccinated. Occurrence of disease even in vaccinated pups is not uncommon and is reported from many countries (Nandi et al., 2010). There are ongoing debates referring to genetic dissimilarity of field viruses and vaccine strains as well as interference between monoclonal antibodies and active immune response. Considering CPVs as highly mutating virus with emerging and re-emerging genetic variants, some studies have suggested that older vaccine strains may not be well protective against current variants (Truyen, 2006; Decaro et al., 2007b; Nandi et al., 2010). Greenwood et al. (1995) demonstrated that CPV-2 vaccine strains are capable to provide protection against variants, CPV-2a and CPV-2b. Pratelli et al. (2001) reported that, upon vaccination with $\mathrm{CPV}-2 \mathrm{~b}$, serum titers in dogs are able to neutralize infection from homologous (CPV-2b) and heterologous (CPV-2, CPV-2a) variants while this may not be the case with dogs vaccinated with CPV-2. On the other hand, Schultz (2008) revealed that current vaccine strains (CPV-2) provide excellent immunity to all genotypes of CPV-2 (e.g. CPV-2a, 2b, and 2c). Larson and Schultz (1997) revealed virus passage and concentration as more important aspect than prevailing variants in inducing protective immune response. Since there is a lack of facilities to perform virus neutralization assay in Pakistan, we were not able to perform virus isolation and subsequent virus neutralization assays using sera from vaccinated dogs. Interference of $\mathrm{mAbs}$ to active immune response is another potential reason for vaccine failure (Buonavoglia et al., 1992; Meers et al., 2007). None of the tested vaccines so far are capable of breaking-through mAbs $(1: 160)$ to yield neutralizing titer regardless of the use of vaccine concentration (Pratelli et al., 2000). Vaccination is likely to be successful if given to seronegative pups or pups with hemagglutination inhibition titer $\leq 10$ (Schultz, 2006). However, titer $\geq 1: 20$ may interfere with active immune response (Meers et al., 2007). As titer $\geq 1: 80$ is considered as protective for 
challenge in field infection, and vaccine can give rise to protective immune response when it is $\leq 1: 10$, there still is a gap where the pup is susceptible for infection. Besides these limitations, another concern is vaccine handling and time of application to dogs (Calderon et al., 2009; Ntafis et al., 2010). Therefore, it is necessary to evaluate antibody titer before vaccination and determine appropriate time for vaccination. After vaccination, it is necessary to monitor the antibody titer of vaccinated dogs.

Although prevalence of other variants cannot be excluded, CPV-2a is appearing to be the most prevalent CPV with high genetic heterogeneity in nucleotide and codon sequence. This differentiation may further increase with the acquisition of more changes in nucleotide and/or codon profile and, thus requires continuous surveillance over a wide geographical area of the country. Since incidence has been reported even in vaccinates, serum evaluation before and after vaccination is recommended to safeguard the pups from CPV.

Acknowledgement. We thank laboratory technicians (Muhammad Ashraf, Muhammad Tariq, Imran Shafique) and supporting staff for help in sample collection and laboratory work.

\section{References}

Agbandje M, Parrish CR, Rossmann MG (1995): The structure of Parvoviruses. Semin. Virol. 6, 299-309. https://doi. org/10.1006/smvy.1995.0036

Aldaz J, Garcia-Diaz J, Calleros L, Sosa K, Iraola G, Marandino A, Hernandez M, Panzera Y, Perez R (2013): High local genetic diversity of canine parvovirus from Ecuador. Vet. Microbiol. 166, 214-219. https://doi.org/10.1016/j. vetmic.2013.06.012

Appel MJ, Scott FW, Carmichael LE (1979): Isolation and immunisation studies of a canine parco-like virus from dogs with haemorrhagic enteritis. Vet. Rec. 105, 156-159. https:// doi.org/10.1136/vr.105.8.156

Buonavoglia C, Martella V, Pratelli A, Tempesta M, Cavalli A, Buonavoglia D, Bozzo G, Elia G, Decaro N, Carmichael L (2001): Evidence for evolution of canine parvovirus type 2 in Italy. J. Gen. Virol. 82, 3021-3025. https://doi. org/10.1099/0022-1317-82-12-3021

Buonavoglia C, Tollis M, Buonavoglia D, Puccini A (1992): Response of pups with maternal derived antibody to modified-live canine parvovirus vaccine. Comp. Immunol. Microbiol. Infect. Dis. 15, 281-283. https://doi. org/10.1016/0147-9571(92)90007-E

Calderon MG, Mattion N, Bucafusco D, Fogel F, Remorini P, LaTorre J (2009): Molecular characterization of canine parvovirus strains in Argentina: Detection of the pathogenic variant CPV2c in vaccinated dogs. J. Virol. Methods 159, 141-145. https://doi.org/10.1016/j.jviromet.2009.03.013

Carmichael LM, Joubert JC, Pollock RV (1980): Hemagglutination by canine parvovirus: serologic studies and diagnostic application. Am. J. Vet. Res. 40, 784-791.
Decaro N, Buonavoglia C (2012): Canine parvovirus - a review of epidemiological and diagnostic aspects, with emphasis on type 2c. Vet. Microbiol. 155, 1-12. https://doi. org/10.1016/j.vetmic.2011.09.007

Decaro N, Desario C, Addie DD, Martella V, Vieira MJ, Elia G, Zicola A, Davis C, Thompson, G, Thiry E, Truyen U, Buonavoglia C (2007a): Molecular epidemiology of canine parvovirus, Europe. Emerg. Infect. Dis. 13, 1222-1224. https://doi.org/10.3201/eid1308.070505

Decaro N, Desario C, Elia G, Campolo M, Lorusso A, Martella V, Bunavoglia C (2007b): Occurrence of severe gastroenteritis in pups after canine parvovirus vaccine administration: a clinical and laboratory diagnosis dilemma. Vac. 25, 1161-1166. https://doi.org/10.1016/j. vaccine.2006.10.020

Decaro N, Desario C, Parisi A, Martella V, Lorusso A, Miccolupo A, Mari V, Colaianni ML, Cavalli A, Di Trani L, Buonavoglia C (2009): Genetic analysis of canine parvovirus type 2c. Virol. 385, 5-10. https://doi.org/10.1016/j. virol.2008.12.016

Greenwood NM, Chalmers SK, Baxendale W, Thompson H (1995): Comparison of isolates of canine parvovirus by restriction enzyme analysis and vaccine efficacy against field strains. Vet. Rec. 136, 63-67. https://doi.org/10.1136/ vr.136.3.63

Hong C, Decaro N, Desario C, Tanner P, Pardo M, Sanchez S, Buonavoglia C, Saliki J (2007): Occurrence of canine parvovirus type C in the United States. J. Vet. Diagn. Invest. 19, 535-539. https://doi.org/10.1177/104063870701900512

Jafri SA, Rabbani M (1999): Prevalence of canine diseases in Lahore area. Pak. Vet. J. 19, 40-42.

Khan MA, Rabbani M, Muhammad K, Murtaza N, Nazir. J (2006): Isolation and characterization of canine parvovirus. Int. J. Agric. Biol. 8, 898-900.

Larson LJ, Schultz RD (1997): Comparison of selected canine vaccines for their ability to induce protective immunity against canine parvovirus infection. Am. J. Vet. Res. 58, 360-363.

Lin CN, Chien CH, Chiou MT, Chueh LL, Hung MY, Hsu HS (2014): Genetic characterization of type 2a canine parvoviruses from Taiwan reveals the emergence of an Ile324 mutation in VP2. Virol. J. 11, 39. https://doi. org/10.1186/1743-422X-11-39

Martella V, Decaro N, Buonavoglia C (2006): Evolution of CPV-2 and implication for antigenic/genetic characterization. Virus Genes 33, 11-13. https://doi.org/10.1007/s11262$\underline{005-0034-8}$

Meers J, Kyaw-Tanner M, Bensink Z, Zwijnenberg R (2007): Genetic analysis of canine parvovirus from dogs in Australia. Australian Vet. J. 85, 392-396. https://doi.org/10.1111/j.1751$\underline{0813.2007 .00206 . \mathrm{x}}$

Maya L, Calleros L, Francia L, Hernandez M, Iraola G, Panzera Y, Sosa K, Perez R (2013): Phylodynamics analysis of canine parvovirus in Uruguay: evidence of two successive invasions by different variants. Arch. Virol. 158, 1133-1141. https://doi.org/10.1007/s00705-012-1591-5

Nandi S, Chidri S, Kumar M, Chauhan RS (2010): Occurrence of canine parvovirus type $2 \mathrm{c}$ in the dogs with haemorrhagic 
enteritis in India. Res. Vet. Sci. 88, 169-171. https://doi. org/10.1016/j.rvsc.2009.05.018

Ntafis V, Xylouri E, Kalli I, Desario C, Mari V, Decaro N, Buonavoglia C (2010): Characterization of canine parvovirus 2 variants circulating in Greece. J. Vet. Diag. Inv. 22, 737-740. https://doi.org/10.1177/104063871002200512

Parrish C, Aquadro C, Strassheim M, Evermann J, Sgro J, Mohammed H (1991): Rapid antigenic-type replacement and DNA sequence evolution of canine parvovirus. J. Virol. 65, 6544-6552.

Pereira CA, Leal ES, Durigon EL (2007): Selective regimen shift and demographic growth increase associated with the emergence of highfitness variants of canine parvovirus. Infect. Genet. Evol. 7, 399-409. https://doi.org/10.1016/j. meegid.2006.03.007

Pereira CA, Monezi TA, Mehnert DU, D'Angelo M, Durigon EL (2000): Molecular characterization of canine parvovirus in Brazil by polymerase chain reaction assay. Vet. Microbiol. 75, 127-133. https://doi.org/10.1016/S0378$\underline{1135(00) 00214-5}$

Perez R, Bianchi P, Calleros L, Francia L, Hernandez M, Maya L, Panzera Y, Sosa K, Zoller S (2012): Recent spreading of a divergent canine parvovirus type $2 \mathrm{a}(\mathrm{CPV}-2 \mathrm{a})$ strain in a CPV-2c homogenous population. Vet. Microbiol. 155, 214-219. https://doi.org/10.1016/j.vetmic.2011.09.017

Perez R, Francia L, Romero V, Maya L, Lopez I, Hernandez M (2007): First detection of canine parvovirus type $C$ in South America. Vet. Microbiol. 124, 147-152. https:// doi.org/10.1016/j.vetmic.2007.04.028

Pratelli A, Cavalli A, Martella V, Tempesta M, Decaro N, Carmichael LE, Buonavoglia C (2001): Canine parvovirus (CPV) vaccination: Comparison of neutralizing antibody responses in pups after inoculation with CPV2 or CPV2b modified live virus vaccine. Clin. Diagn. Lab. Immunol. 8, 612-615. https://doi.org/10.1128/cdli.8.3.612-615.2001
Pratelli A, Cavalli A, Normanno G, De Palma MG, Pastorelli G, Martella V, Buonavoglia C (2000): Immunization of pups with maternally derived antibodies to canine parvovirus (CPV) using a modified-live variant (CPV-2b). J. Vet. Med. B, Infect. Dis.Vet. Public. Health. 47, 273-276. https://doi.org/10.1046/j.1439-0450.2000.00340.x

Reed AP, Jones EV, Miller TJ (1988): Nucleotide sequence and genome organization of canine parvovirus. J. Virol. 62, 266-276.

Schultz RD (2006): Duration of immunity for canine and feline vaccines: a review. Vet. Microbiol. 117, 75-79. https:// doi.org/10.1016/j.vetmic.2006.04.013

Schultz RL (2008): Current canine parvovirus type 2 (CPV-2) vaccines provide excellent immunity to all genotypes of CPV-2 (e.g. CPV- 2a, 2b, and 2c). Vet. Ther. 9, 94-101.

Shackelton LA, Parrish CR, Truyen U, Holmes EC (2005): High rate of viral evolution associated with the emergence of carnivore parvovirus. Proc. Natl. Acad. Sci. USA 102, 379-384. https://doi.org/10.1073/pnas.0406765102

Towakal F, Rabbani M, Muhammad K, Khan MS, Shabbir MZ (2010): Major strains of canine parvovirus present in dog population of Pakistan. Pakistan J. Zool. 42, 833-836.

Truyen U (2006): Evolution of canine parvovirus - a need for new vaccines? Vet. Microbiol. 117, 9-13. https://doi. org/10.1016/j.vetmic.2006.04.003

Truyen U, Evermann JF, Vieler E, Parrish CR (1996): Evolution of canine parvovirus involved loss and gain of feline host range. Virol. 215, 186-189. https://doi.org/10.1006/ viro.1996.0021

Umar S, Ali A, Younus M, Maan MK, Ali S, Khan WA, Irfan M (2015): Prevalence of canine parvovirus infection at different pet clinics in Lahore, Pakistan. Pakistan J. Zool. 47, 657-663.

Zhao H, Wang J, Jiang Y, Cheng Y, Lin P, Zhu H, Han G, Yi L, Zhang S, Guo L, Cheng S (2015): Typing of canine parvovirus strains circulating in North-East China. Transbound. Emerg. Dis. https://doi.org/10.1111/tbed.12390 